Si aceptamos la idea de los neorretóricos de la Retórica como disciplina general del discurso, podemos describir la traducción como una operación retórica. En la traducción de poesía esto es particularmente notable por ser un género muy sistematizado. Al analizarla podemos hacer uso, pues, de categorias retóricas tradicionales. Incluso podemos encontrar diferencias de estrategia traductora entre categorias tan próximas en apariencia como la dispositio y la compositio. Como muestra, analizamos una traducción colectiva de un fragmento de un conocido poema del autor turco A. H. Tanpınar.

PALABRAS CLAVE: Retórica, traducción de poesía, dispositio, elocutio, traducción turco-español.

\title{
Dispositio y compositio en la traducción de poesía turco-español
}

Rafael Carpintero

Universidad de Estambul

\section{Dispositio and compositio in translation of poetry Turkish-Spanish}

If we accept the conception of Rhetoric as a general discipline of discourse as held by the New Rhetoricians, we could describe translation as a rhetorical operation. This is particularly evident in the translation of poetry, being a highly systematized genre. Thus, we can make use of traditional rhetorical categories when analyzing poetry translations. We can even find translation strategy differences between categories so close in appearance as the dispositio and the compositio. As an example, we analyze the collective translation of a fragment of a well-known poem by the Turkish author A. H. Tanpınar.

KEY WORDS: rhetoric, poetry translation, dispositio, elocutio, turkish-spanish translation. 
es de sobra conocida la frase atribuida a Robert Frost de que la poesía es lo que se pierde en las traducciones (poetry is what gets lost in trans100 lation), pero también está bastante extendida, sobre todo entre traductores, la opinión contraria: que la poesía es, precisamente, lo que permanece cuando se traduce. En ambos casos es evidente una concepción casi mágica de lo poético: la poesía es lo inaprehensible y lo inefable y por lo tanto es intraducible; o bien, la poesía reside en lo más profundo del alma humana y por lo tanto permanece sea cual sea la lengua en la que se expresa.

En cualquier caso, es un hecho que la poesía se traduce, mejor o peor. Es también un hecho que lo que se debate habitualmente no es tanto la posibilidad de lograrlo sino la manera de traducirla. Y esto nos permite plantearnos la pregunta fundamental: ¿Qué es lo que diferencia a la poesía de otros géneros para que sea tan polémica su traducción? Para responderla primero tendremos que ponernos de acuerdo en qué es lo que entendemos por poesía desde el punto de vista de su traducción.

\section{QUÉ ENTENDEMOS POR TEXTO POÉTICO Y QUÉ PROBLEMAS PLANTEA SU TRADUCCIÓN}

Por lo general, cuando se habla de poesía, se habla de verso. De hecho, en el lenguaje corriente es habitual que ambos términos sean casi sinónimos (Torre, 2000/2014: I3-I4). Visto así, la poesía sería lo opuesto a la prosa. Sin embargo, podemos encontrarnos muchos géneros o subgéneros mestizos o fronterizos. Se habla de poesía en prosa, por ejemplo, como una especie de verso libre llevado al extremo. En este caso, la poesía residiría más en la sustancia que en la forma, si usamos la terminología de Hjelmslev (Prolegómenos a una teoría del lenguaje, I943), lo que quizá le quitara la razón a Robert Frost. Por otra parte tenemos toda la tradición del teatro clásico en verso, por ejemplo, que no en todo momento puede ser calificado de poesía; así como las formas más narrativas, como las epopeyas e incluso muchas fábulas.

Para nuestro propósito, que es el de reflexionar sobre algunas posibles formas de traducción de la poesía, vamos a mantenernos dentro de los límites de lo que se acepta habitualmente como tal; es decir, el género lírico, casi todo el épico y solo algunos fragmentos del dramático, y siempre en verso. Si excluimos parcialmente las obras épicas es porque son bastante más susceptibles de ser traducidas en prosa al ser esencialmente narrativas. Por su parte, los fragmentos de obras de teatro que pueden ser vistos como poemas en sí mismos, tienen la misma consideración que un poema aislado y, por lo tanto, no es necesario contemplar la obra íntegra ni analizarlos como muestras representativas del género dramático.

El problema de la traducción de poesía es doble. Por un lado se trata de una forma literaria en la que predomina la economía en la expresión, rica en connotaciones. O dicho de otra forma, con un mínimo de palabras se consiguen un máximo de significaciones y sentidos secundarios, lo que posibilita múltiples lecturas. Por otro, en la poesía - la poesía bien hecha- se produce tal fusión de fondo y forma, tal integración entre contenido y expresión, que sería conveniente conservar dicha forma al traducirla. Como por lo general no es posible mantener en el mismo grado ninguna de ambas cosas - ni las connotaciones, ni la forma de la expresión-, se procura buscar algún tipo de equivalencia que consiga que el texto traducido funcione como el original en la cultura de llegada. Esta intención de conseguir en el lector de la traducción un efecto similar al que produjo el poema en los lectores originales ha llevado a numerosos 
experimentos que, a pesar de su valor, dudosamente pueden ser calificados de traducción en un sentido tradicional. Como ejemplo podemos citar algunas traducciones al inglés de poemas de Catulo como las que mencionan André Lefevere (I992: I25-I37) o Susan Bassnett (I980: 83-92). De igual manera que es discutible considerar estos experimentos ${ }^{\mathrm{I}}$ como traducciones en $^{-}$ un sentido estricto, también es difícil saber si el efecto que produce en el lector actual la lectura de estas versiones es similar al de los poemas originales de Catulo en los lectores de su época.

A la fusión entre fondo y forma común a toda obra literaria, pero que se produce de forma especial en poesía o, al menos, en el verso, es a lo que se refería Jakobson cuando dijo aquella famosa frase sobre la función poética: "The poetic function projects the principle of equivalence from the axis of selection into the axis of combination. Equivalence is promoted to the constitutive device of the sequence» (1958: 358). Hay que entender la frase teniendo en cuenta que Jakobson está hablando de la función poética como una más de las funciones del lenguaje, que no es exclusiva de la poesía ni es la única que se manifiesta en ella — aunque sí sea la principal-: «Any attempt to reduce the sphere of poetic function to poetry or to confine poetry to poetic function would be a delusive oversimplification» (Jakobson, I958: 356). La idea de la que parte Jakobson es que al construir cualquier enunciado podemos escoger entre elementos equivalentes dentro del mismo eje paradigmático. Él propone un ejemplo léxicosemántico - elegir entre child, kid, youngster,

\footnotetext{
I Llevados incluso al ámbito escolar, como puede verse en «From Literal to Literary: A Translation Project for Latin Poetry Classes», un interesante artículo de tres profesores de la Universidad de Iowa (Lindgren, Blumberg and Langseth, 20Io).
}

etc.- , pero podríamos utilizar cualquier otro nivel —el del paradigma verbal es quizá el más claro- y siempre estaríamos moviéndonos en ese eje que Saussure llamaba «en ausencia» por la sencilla razón de que únicamente podemos utilizar uno de esos elementos en el enunciado. No obstante, en un texto en el que predomine la función poética, estas equivalencias se manifiestan también a lo largo del eje sintagmático; es decir, continuamente van apareciendo elementos cuya repetición nos hace ser conscientes de la forma que adopta el mensaje. En la poesía el grado de redundancia es extraordinariamente alto. Los ejemplos más evidentes, como dice el propio Jakobson, son el metro y la rima, pero podemos incluir efectos sonoros como la aliteración y las analogías semánticas que forman la base de gran parte de los tropos. Sin ir más lejos, la metáfora se basa precisamente en esta proyección del eje paradigmático en el sintagmático creando equivalencias entre dos elementos en los que es posible encontrar aspectos comunes basados en una relación de parecido.

Esta proyección del eje de selección sobre el de combinación aumenta las connotaciones posibles y es francamente difícil de mantener en la traducción. Entre otros motivos porque también puede cumplir una función prácticamente opuesta, la de «anclaje», como lo llamaría Roland Barthes («Rhétorique de l'image», I964: 44), al limitar el significado denotativo de algunos términos. Dicho de otra manera, las isotopías que va desplegando el cotexto - el entorno o marco textual - limitan los posibles significados «de diccionario» de las palabras, sin que eso implique que en textos poliisotópicos, como son los poéticos, las connotaciones se reduzcan a una lectura única.

Veamos un ejemplo muy sencillo de Juan Ramón Jiménez que puede aclarar un poco el 
tema y que al mismo tiempo plantea un interesante problema de traducción. Se titula «El poema» y dice:

102

\section{¡No le toques ya más, que asi es la rosa!}

El tema principal de estos dos versos nos viene dado por el título, gracias al cual sabemos que tratan sobre el poema en sí mismo y que, por lo tanto, tenemos que interpretar el pronombre masculino de complemento directo «le» - que sea un leísmo es otra cuestión- como sustituyendo a "poema». Pero el segundo verso incluye un elemento que genera una segunda isotopía, la «rosa», que se relaciona directamente también con «no tocar». Así se crea una correspondencia directa entre «poema» $\mathrm{y}$ «rosa» a través del pronombre «le». Al mismo tiempo, la combinación de los elementos limita las posibles interpretaciones de la rosa como símbolo - como signo, en sumaa las de «naturaleza» y "perfección». Como dice Juan Eduardo Cirlot en su Diccionario de símbolos: «La rosa única es, esencialmente, un símbolo de finalidad, de logro absoluto y de perfección» (Cirlot, I968: 390). Otros sentidos de la rosa como pueden ser el de símbolo de la pasión o el color rojo - recordemos aquel verso de Garcilaso de «En tanto que de rosa y de azucena...»- no son pertinentes gracias a ese «no tocar» que reduce la flor a ser símbolo de la perfección natural frente a lo manipulado $y$, por tanto, artificial. El poema es como la rosa y la rosa es naturalmente perfecta. ${ }^{2}$ La proyección del eje de selección en el eje de combinación ha producido unas equivalencias —en este caso entre «poema» $\mathrm{y}$ «rosa»- que, a través de una

2 Hay quien le da al poema el sentido opuesto considerando la rosa un ejemplo de flor creada artificialmente al contrario que, por ejemplo, las margaritas o las amapolas. No obstante, parece una interpretación bastante retorcida. limitación de los significados posibles de cada uno de los elementos, enriquecen el significado general de la obra cuando se relacionan entre sí.

Sin embargo, este mínimo poema es citado con frecuencia de forma errónea. En muchas ocasiones podemos encontrar que el pronombre masculino «le» aparece en femenino - «la»con lo que estaría hablando únicamente de la rosa. Cabe preguntarse si no ocurriría lo mismo al traducirlo a una lengua como el inglés que tendría que usar el pronombre neutro «it» para ambas cosas; o al turco, que directamente carece de género gramatical. ${ }^{3}$

Por otra parte, en poesía, en la literatura en verso en general, tienen importancia también otros factores como la rima y el metro - es decir, la medida y tipo de verso - Sin embargo, como la traducción supone la trasposición de unos términos —en la lengua terminal - por otros - los de la lengua original- que nunca son del todo equivalentes, eso implica que las estructuras formales muy probablemente no podrán mantenerse, aunque simplemente sea porque las palabras tengan una longitud distinta y acaben, y por lo tanto rimen, de forma diferente. Con todo, precisamente en eso consiste la esencia del verso, en unas medidas, en unos ritmos, en unas rimas.

\section{POÉTICA Y RETÓRICA}

En su libro Retórica general, el Grupo $\mu$ de Lieja prefiere llamar «retórica» a la función "poética» de Jakobson ya que, afirman, se refiere a la expresión de todos los enunciados y no única-

3 Un problema que puede llegar a ser bastante serio. Por ejemplo, en la traducción española de La nube enamorada de Nâzım Hikmet (trad. de Fernando García Burillo y Metin Karadağ), «nube» es palabra necesariamente femenina, mientras que en el texto queda bastante claro que se trata de una nube varón. 
mente los literarios (Grupo $\mu$, I970: 6r). Según los neorretóricos en general habría que considerar la poética, entendida como disciplina de la literatura, dentro del marco general de la retórica como disciplina de la expresión. Durante muchos siglos ha sido justamente al contrario porque se entendía la retórica únicamente como el estudio de las figuras.

La consideración de la Retórica como ciencia global del discurso es la postura de algunos neorretóricos españoles como Tomás Albaladejo y Antonio García Berrio (vid. 1984: 7-59). Hasta el punto de que en un artículo Albaladejo asimila la inventio y la dispositio a la división de la semiótica en sintaxis, semántica y pragmática que hizo Charles Morris en su Fundamentos de la teoría de los signos (1938: 34-36). Según Albaladejo, a la inventio le corresponderían los aspectos semánticos, a la dispositio los sintácticos, y ambas compartirían los pragmáticos (1988-I989: II). La elocutio, como expresión que es -Hjelmslev la llamaría «forma de la expresión»-, incluiría los aspectos semántico, sintáctico y pragmático, pero a nivel de estructuras superficiales.

Tanto la inventio como la dispositio comparten aspectos verdaderamente esenciales ya que no debemos olvidar que son formantes del discurso junto con la elocutio. Es decir, ninguna de las tres partes clásicas de la retórica debe ser vista de manera aislada, sino que todas se entrecruzan en la formación del discurso. Es en este sentido como conviene regresar a la afirmación de Jakobson sobre la función poética. Dice Albaladejo:

De acuerdo con esta distinción entiendo que la inventio y por consiguiente la estructura de conjunto referencial del texto retórico son, respectivamente, una operación y un constructo fundamentalmente semántico- extensionales, ${ }^{4}$ lo cual no está en contradicción con que posean una organización sintáctica: los seres, estados, procesos y acciones que produce la inventio, si bien son elementos semántico-extensionales, están sintácticamente organizados, pues una construcción semántica no puede sostenerse sin una estructuración sintáctica. ${ }^{5}$ Por otro lado, la dispositio y la macroestructura textual son, respectivamente, una operación y un constructo de índole sintáctica que poseen una dimensión secundaria semántico-extensional, ya que la serie de relaciones de dispositio no puede establecerse sobre el vacío, sino que por el contrario está construida sobre elementos semánticos como son los loci. (Albaladejo Mayordomo, I988-I989: I2)

Si interpretamos esta afirmación en términos de la función poética de Jakobson, podemos ver que la operación de selección en el eje paradigmático que supone la inventio en el plano semántico —elegir «rosa», por ejemplo, en lugar de «clavel»— se organiza sintácticamente en el eje de combinación, dominio de la dispositio, construida a su vez sobre los loci, esos «lugares» donde la inventio busca las ideas; los símbolos florales en el caso del poema de Juan Ramón Jiménez. Se produce así un movimiento helicoidal con el que inventio y dispositio se enriquecen

4 Siguiendo una división tradicional que asocia inventio a res - al referente, según él-y elocutio a verba - la forma de la expresión, en suma-, Albaladejo distingue entre «una res extensional, de índole referencial y dependiente de la inventio, y una res intensional, de carácter cotextual y vinculada a la dispositio como operación por la que se organizan dentro del discurso las ideas encontradas por medio de la inventio» (Albaladejo Mayordomo, I988-I989: Io). Es decir, lo extensional se relaciona con el referente (externo) y lo intensional con el texto. También podríamos verlos respectivamente como los ámbitos del contexto y del cotexto.

5 Debemos entender «sintáctico» en el sentido saussuriano de relaciones entre los elementos presentes en una cadena. Puede ser, por lo tanto, en un nivel superior o inferior al de la frase, dominio de lo que habitualmente entendemos por sintaxis. 
mutuamente, sobre todo en la poesía. Pero, ¿qué ocurre cuando hay que traducir este tipo de textos que funden de una manera tan intensa la 104 forma y el contenido?

\section{ESTRATEGIAS DE TRADUCCIÓN POÉTICA: LEFEVERE Y HOLMES}

La imposibilidad de traducir poesía de la que hablaba Frost reside en que el simple cambio de lengua dificulta enormemente todo lo que se pone en funcionamiento en la expresión en verso, desde el metro en sí a la gran densidad connotativa. Además se espera que la traducción no solo sea adecuada al original en todos los niveles, sino que también sea aceptable en la lengua de destino como un poema en sí misma:

[...] in addition to the difficulties involved in accounting for content and form, sounds and associations, the translator of poetry is also often expected to produce a text that will function as a poem in the TL. (Connolly, 1998/200I: 171).

Lo problemático del proceso ha generado una gran cantidad de estrategias - ninguna de ellas óptima- o de descripciones de traducciones ya hechas, a ser posible, por autores de cierto renombre.

The view that it is impossible to translate poetry recognizes that it is impossible to account for all the factors involved and to convey all the features of the original in a language and form acceptable to the target language culture and tradition. However, from this sobering acceptance of the difficulty involved and of the enormity of the task comes a search for strategies whereby as much as possible of the original poetry may be saved in the translation. (Connolly, ibid.)
A partir del eterno debate de si se debe traducir la poesía en verso o en prosa, podemos encontrarnos propuestas que oscilan entre el máximo respeto posible al contenido o bien a la forma, entendiendo por tal la forma métrica. Entre las estrategias descritas, nos interesan especialmente la clasificación de Lefevere (Translating Poetry: Seven Strategies and a Blueprint, 1975) y, sobre todo, la de Holmes («Forms of Verse Translation and the Translation of Verse Form», I970).

E1 libro de Lefevere parece haber tenido especial fortuna y existen diversos trabajos académicos que aplican su «modelo», ${ }^{6}$ que en realidad no es sino una categorización de distintas soluciones a los problemas que plantea traducir el poema número 64 de Catulo (vid. Bassnett, I980: 83-85). Lefevere distingue siete estrategias: (I) la traducción fonética, que trata de mantener el sonido del original; (2) la literal, palabra por palabra; (3) la métrica, que preserva el metro del original; (4) la traducción en prosa; (5) la rimada, con metro y rima; (6) en verso blanco; y (7) lo que Lefevere llama «interpretación», que va desde la «versión» en la que se prescinde de la forma del original, a la «imitación», en la que el original es únicamente un pretexto para la creación de un poema nuevo. Este último procedimiento, al menos eso se suele asegurar, pretende mantener la intención pragmática del

6 Una búsqueda rápida en internet nos ofrece dos estudios recientes escritos en Irán: Kolahi, Sholeh y Mahgol Emamian Shiraz (20I2): «Application of Lefevere's Seven Strategies in English Translations of Sohrab Sepehri's Poems». International Journal of Linguistics, Vol. 4 , No ${ }_{4}$, 450-467; Sharif, Forouzan Dehbashi y Ramin Yarmohamadi Khameneh (2015): «A Model for Translating Poetry Based on the Lefevere's Theory on Poetry Translation and Dastjerdi's Model». ELT Voices-International Journal for Teachers of English, Vol. 5, No 4, 53-65. Por conversaciones con colegas me consta que se trata de un ejercicio bastante popular en las clases de traducción (de poesía) intentar las siete estrategias descritas por Lefevere. 
original para producir el mismo efecto en el lector actual, salvando distancias temporales y geográficas.

Podemos ver que todas las estrategias descritas por Lefevere pueden ir agrupándose en pares. Por una parte, la traducción más «tradicional» que mantiene una mayor o menor fidelidad con el original y por otra la «interpretación». Por un lado, las traducciones en prosa, incluyendo la literal, _que supuestamente cubren todos los significados posibles al no estar sometidas a restricciones métricas- y por otro las traducciones en verso. Dentro de estas últimas cabría también distinguir entre las que quieren mantener cierta similitud con el original - la fonética y la métrica- o al menos una relativa disciplina estrófica - la rimada-y las que, aun en verso, adoptan una forma más libre - en verso blanco-. Este último caso, sin embargo, es altamente discutible para lenguas que no sean el inglés. En efecto, mientras que el verso blanco es bastante común en la literatura inglesa, no ocurre lo mismo en otras tradiciones, como la castellana, en la que el sistema se fundamenta más en la cantidad silábica y no tanto en la distribución de los acentos. Es la diferencia que se ha establecido entre lenguas de las llamadas stress-timed y syllable-timed. En las primeras, de una mayor regularidad acentual, la base rítmica es la isocronía acentual, es decir «la hipotética igualdad de las distancias temporales entre los acentos» (Torre, 2003: 274), mientras que en las segundas lo fundamental es la cantidad silábica.

Para Almeida (1994),7 habría que hablar de lenguas de ritmo acentual, con isocronía localizada en el pie, como en las lenguas germánicas, y lenguas de ritmo silábico, con isocronía localizada en la sílaba, como en la

7 Almedia, M.: «Patrones rítmicos del español. Isocronía y alternancia», Estudios Filológicos, 29, I994, pp. II8-I23. mayoría de las lenguas románicas. Al primer grupo, caracterizado por la recurrencia acentual con distancias similares, se adscriben, en fin, lenguas tan dispares como el inglés, el ruso y el árabe. Al segundo grupo, definido por la similitud temporal entre las sílabas, se asignó el español, junto con otras lenguas romances, como el italiano, el francés y el catalán. (Torre, 2003: 275)

En cualquier caso, se trata solo de tendencias, y muy discutibles como subraya el mismo Torre, puesto que en ambos grupos de lenguas - $\mathrm{O}$ al menos en inglés y en español- existen tanto ritmos acentuales como regularidad silábica en los versos. Con todo, sí es cierto que en inglés es relativamente fácil mantener los pies acentuales mientras que no lo es tanto en español, como lo demuestra el que ellos llamen iambic pentameter a lo que nosotros llamamos «endecasílabo». Que a un verso tan fundamental se le denomine según el nombre del patrón rítmico deja bastante clara la posibilidad de las composiciones en verso blanco; en cambio, en español existen múltiples clases de endecasílabo según la posición de los acentos y la combinación de distintos tipos en el mismo poema no se considera necesariamente algo negativo.

A la hora de describir las posibilidades de la traducción de poesía parece más útil la división de Holmes en cuatro tipos (I970). De entrada, Holmes descarta las traducciones en prosa -incluyendo las literales de cualquier clase- porque únicamente la traducción en verso «manifestly aspires to be a poem in its own right» (I970: 93). Dentro de las escritas en verso, observa cuatro tipos generales. El cuarto, que llama deviant o extraneous form corresponde a las «interpretaciones» de Lefevere, o sea, a obras que toman el original como base para construir algo distinto. Las tres posibilidades restantes - las que podríamos llamar traduc- 
ciones en verso propiamente dichas - son (a) la «forma mimética» en la que se retiene la forma métrica del original —el hexámetro, por ejemplo, o el haiku—; (b) la «forma analógica», que busca una forma paralela a la original en la propia tradición literaria - la octava real para los poemas épicos—; y (c) la «forma orgánica», en la que el traductor mantiene el «material semántico» dejando que el poema vaya desarrollando su forma según avanza la traducción (1970: 95-97). Los dos primeros tipos los considera «form derivative» ya que pretenden mantener ciertas estructuras formales, mientras que el tercero es «content derivative» porque se centra en mantener el contenido. Este último sistema, como reconoce Holmes, es el más seguido en la actualidad.

\section{ESTRATEGIAS DE TRADUCCIÓN Y COMPONENTES DEL DISCURSO}

Podemos relacionar con bastante facilidad estas categorías de Holmes con las partes principales de la retórica tal y como las interpretan los neorretóricos. Tanto las traducciones en prosa como las «deviant/extraneous» respetan fundamentalmente la inventio, es decir, las categorías semánticas que constituyen el conjunto referencial del texto. ${ }^{8}$ Las "form derivative» (la analógica y, sobre todo, la mimética) se basan en la dispositio del original. Y las «content derivative» se centran en la elocutio porque tienden a mantener en mayor grado que las anteriores figuras no únicamente semánticas sino también fonéticas y morfosintácticas.

8 Según Stefano Arduini (Prolegómenos a una teoría general de las figuras, 2000: 7I), la operación previa de la intellectio "construye el modelo retórico de mundo que constituirá el material para el texto retórico». La inventio, por su parte, seleccionará los loci, los lugares, pertinentes dentro de ese mundo para ir edificando el texto.

El soneto y el haiku nos ofrecen dos magníficos ejemplos en lo que respecta a la dispositio en la traducción mimética. En el primero, la tradición castellana recogió la forma italiana de dos cuartetos y dos tercetos en la que en el primer cuarteto se plantea una situación que se desarrolla o amplía en el segundo, el primer terceto resuelve o comenta el asunto planteado en los cuartetos y el segundo terceto expresa una reflexión general sobre el tema. En el haiku tiene que mantenerse un esquema de 5-7-5 sílabas y debería incluir una emoción producida por la contemplación de la naturaleza y una referencia a la estación del año (kigo). Tanto el soneto como el haiku, aunque el primero más, obligan a una disposición particular de las ideas que van a expresarse. Cómo se lleva a cabo exactamente, es decir, qué léxico, qué figuras retóricas o qué rima concreta se va a emplear, corresponde a la elocutio. Siguiendo con esta idea podemos considerar que los aspectos formales de la traducción analógica - en la que se busca un correspondiente a la forma original en la propia tradición - pertenecen asimismo al campo de la compositio, es decir, de la elocutio, ya que tenderá a respetarse la estructura semántica del original y las cuestiones de medida y rima se convertirán en un problema de expresión y no de disposición del contenido.

Otra de las ventajas que aporta el «sistema» de Holmes es la idea del poema traducido como «metapoema», dentro del concepto general de la traducción de literatura como «meta-literatura» (Holmes, I970: 93). Y es una ventaja porque si concebimos la traducción en general como una operación retórica (vid. Carpintero, 20I7), esta denominación encaja perfectamente con la de las figuras retóricas como metáboles que hace el Grupo $\mu$ en su Retórica general: «Llamaremos metábole a toda clase de cambio de cualquier aspecto del lenguaje» (I970: 62); un «cambio de 
aspecto» bastante obvio en el caso de la traducción.

Las metáboles se generan en cada uno de los niveles lingüístico-textuales - fonético-morfológico, sintáctico, semántico, lógico-textualsegún cuatro operaciones básicas: (a) supresión; (b) adjunción; (c) supresión/adjunción —llamada también «substitución» en el Tratado del signo visual (Grupo $\mu$, 1992: 139 y 351-353)—; y (d) permutación (Retórica general, 1970: 91-95). En este sentido cabría analizar las soluciones descritas por Holmes como supresión del contenido o de la forma - según se priorice lo contrario en las formas «mimética» $\mathrm{y}$ «orgánica»—; como adjunción de una «forma analógica» al contenido original; o como permutación en la forma «desviada» o «ajena». Muy posiblemente, en las formas mimética y analógica se produzcan también múltiples y casi inevitables sustituciones de unos elementos por otros al ajustar el contenido traducido a unas formas preexistentes.

La producción de un metapoema traducido implica inevitables metaplasmos - figuras de dicción - relacionados directamente con la elocutio simplemente por el hecho de que ni las palabras ni su morfología son iguales en las dos lenguas y no «suenan» de forma parecida; metasememas y metalogismos - figuras semánticas y de lógica textual, respectivamente- porque las lenguas no organizan de igual manera el universo ni su expresión y, por lo tanto, poseen distintos loci para la inventio; pero también implican metataxis -alteraciones en la sintaxis-, bien por motivos estrictamente sintácticos del orden natural de la frase, bien para ajustar el contenido a la forma métrica.

Estas metataxis pertenecen a lo que tradicionalmente se ha llamado compositio, parte de la elocutio que, según Barthes, es el «campo asociativo de las palabras en la oración» (1970/1985: 158). Con todo, al traducir es posible distinguir entre los cambios a los que obliga la sintaxis propia de cada lengua, y que desde el punto de vista de la traducción sí corresponden a fenómenos propios de la compositio como parte de la elocutio - por ejemplo, la posición del adjetivo con respecto al sustantivo-, de los desplazamientos de distintos elementos en el interior del poema concebido como obra completa, y que podrían considerarse dentro de la dispositio - por ejemplo, el distinto orden de los versos aunque se deba a motivos gramaticales- $-\mathrm{O}$ incluso una distinta apreciación de un mismo fenómeno según se haga desde un punto de vista sintáctico o semántico.

Existen lenguas cuya sintaxis es radicalmente distinta, como el español y el turco, por lo que las traducciones requieren enormes ajustes en el nivel oracional. Cuando se traduce del turco al español, el paso de una lengua altaica — aglutinante- a otra románica implica unas desviaciones ${ }^{9}$ sintácticas inevitables en la compositio de la oración, especialmente en lo que se refiere a la posición del verbo y a la de los calificadores y determinantes del sustantivo — sobre todo adjetivos, pero también otros mucho más complejos, incluyendo frases subordinadas-, así como al ordenamiento de lo que en turco se expresa mediante una única palabra pero que en español requiere sintagmas o incluso frases completas. Pero cuando se traduce poesía, además de esto se producen alteraciones del orden natural de la oración cuando intentamos ajustarnos a unos patrones métricos que, por su propia naturaleza, no son nada elásticos. Esto, que en principio es independiente de la lengua de la que se traduzca porque solo debería afectar al resultado en la

9 Aunque no consideremos como «desviaciones» (shifts) ni las figuras retóricas ni la traducción, tampoco podemos negar que esta última es una forma distinta de un original preexistente. En traducción sí que existe un verdadero «grado cero», que es el original. 
lengua de llegada, se acentúa según sea más lejana la lengua original porque el texto de partida también está sometido a unos patrones métricos y rítmicos que están pensados precisamente para esa lengua.

Encontramos un buen ejemplo en los hipérbatos. En turco la llamada devrik cümle, la «frase invertida», ya de por sí le concede un aire poético al texto, hasta el punto de que es una de las tácticas habituales al traducir poesía. Sin embargo, esta devrik cümle, al contrario de lo que ocurre en castellano, suele ser más próxima al habla oral y, por lo tanto, más fácil de entender. ¿Cómo puede solucionarse esta figura al traducir al español? ¿Cómo podemos invertir el orden de, por ejemplo, poseído y poseedor sin resultar artificiosos, justo al contrario que en el original? Sin olvidar que en muchas ocasiones el hipérbaton es un recurso utilizado en el original para ajustar el ritmo o la rima y que no puede ser reproducido en la traducción con una fidelidad absoluta, aunque solo sea porque las palabras tienen sonidos distintos en ambas lenguas. En suma, es difícil, si no imposible, reproducir el hipérbaton de «de su dueña tal vez olvidada» si queremos mantener el efecto y además pretendemos que rime con «veíase el arpa». ${ }^{\text {. }}$

io De los dos traductores al turco de este conocido poema de Bécquer, Ayşe Nihal Akbulut mantiene el hipérbaton del primer verso alterando el orden natural del genitivo turco («Karanlık bir köşesinde salonun» * Oscuro un ángulo-en salón-del») mientras que Ertuğrul Önalp preserva, relativamente, el orden de la frase en español y el natural de la frase en turco ( «Salonun karanlik bir köşesinde» "«Salón-del oscuro un ángulo-en»). Ambas soluciones se sitúan en el ámbito de la elocutio, respetando o no el orden original de la frase o bien el hipérbaton como figura. En la estrofa en su totalidad podemos observar, en cambio, una clara estrategia dispositiva - de la dispositioen cómo Akbulut deja al final la palabra «arpa», aunque no rime con nada, mientras que Önalp no lo hace, respetando el orden sintáctico turco. A pesar de que el resultado pueda parecer similar, creo que se trata de estrategias pensadas en niveles totalmente distintos.
En un artículo publicado en Target ( On Aboriginal Sufferance: A Process Model of Poetic Translating», 1989), Francis R. Jones propone un interesante sistema de traducción de poesía con el que pretende salvar hasta cierto punto el dilema de sacrificar unos aspectos en favor de otros. Su sistema se divide en tres fases: comprensión, interpretación y creación, aunque nos vamos a detener en particular en la primera y la segunda. Para proceder plenamente a la fase de «interpretación», y con el objetivo de aplicar determinadas técnicas de traducción, primero el traductor «sopesa» el valor relativo de los distintos componentes del poema:

What I suggest is that the translator, either at the initial analysis or, more usually, once he or she meets with specific problems later on, gives the structures I have mentioned some sort of relative weighting - e.g. "the sound-structure is absolutely vital, the exact word-meaning less so". (Jones, I989: I90)

Este «sopesado» debe hacerse en función de lo que, haciendo una analogía con la química, Jones llama «valencias» de los elementos; es decir, las distintas posibilidades combinatorias que ofrecen. Y lo importante es que algunas valencias pueden tener más peso relativo que otras:

Examples of valent features might be an item's literal meaning, associative meaning, etymological meaning, pragmatic meaning; its concrete or metaphorical role in the image; its typical collocations and its actual collocations in this text; repetitions elsewhere in the text; style, register; sound-quality/length; syntactic function, morphological form; and so on. Moreover, because the textual networks have been assigned, whether intuitively or consciously, distinct weightings in terms of their importance to the image or to the text 
as a whole, some valent features are more important than others. (Jones, I989: I90)

Las soluciones que ofrece a los problemas de traducción son varias, pero lo fundamental es este sistema de «valencias», de posibilidades de combinación en el plano sintagmático que permiten decidirse por una forma u otra - del eje paradigmático-, aunque sea de forma intuitiva y subjetiva, como bien reconoce Jones: «the subjetivities of understanding and recreativity [...] become central to the task of translation» [en poesía] (1989: 195). El ejemplo más sencillo quizá lo constituya el sistema de rimas, en el que lo fundamental es mantener un sonido, pero que también implica relacionar de forma casi inevitable las palabras que riman entre sí: «De la repetición sonora, surge necesariamente una cierta relación semántica, por asociación o por contraste, entre las palabras enlazadas por la rima» (Torre, 2000/20I4: 57). Del «sopesado» por parte del traductor de las «valencias» de cada uno de los términos posibles dependerá la decisión de mantener la rima, aún a coste del ripio, o la relación semántica entre las palabras que riman en el original.

\section{Un ejemplo: el taller de traducción de TEDA}

Vamos a ver un ejemplo de estas diferencias entre soluciones relativas a la compositio debidas a motivos morfosintácticos, pero también de medida o rima; o a la dispositio, por la disposición de los elementos en el texto. Se trata de la traducción del primer cuarteto del que es quizá el poema más famoso de Ahmet Hamdi

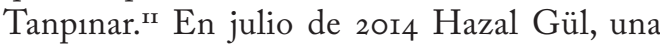

\footnotetext{
II Ne içindeyim zamanın, / Ne de büsbütün dışında; / Yekpâre, geniş bir ânın / Parçalanmaz akışında. Tanpınar (IgOI-I962) es uno de los grandes clásicos contemporáneos de la literatura turca y el maestro indiscutible del Nobel
}

antigua estudiante del departamento de español de la Universidad de Estambul, se puso en contacto con el grupo formado por los participantes en los primeros talleres de traducción españolturco y turco-español organizados en Estambul por el programa TEDA ${ }^{\mathrm{I} 2}$ del Ministerio de Cultura turco con la intención de pedir opinión sobre su traducción de los primeros cuatro versos de dicho poema. ${ }^{\mathrm{I} 3} \mathrm{Su}$ propuesta generó un interesante debate, sobre todo en torno a los versos tercero y cuarto. ${ }^{\text {I4 }}$

Los dos primeros no provocaron mayor discusión, a pesar de que planteaban un interesante problema. En ellos existe una antítesis entre «dentro» $y$ «fuera» que se establece mediante un paralelismo sintáctico inevitable ya que se trata de una oración distributiva («ni... ni...»). Sin embargo, la posición de cada uno de estos elementos en el verso correspondiente es distinta en el original, posiblemente por motivos de rima. El «dentro» se sitúa en el centro del primer verso, mientras que el «fuera» está al final del segundo. De hecho, el primer verso es una devrik cümle ya que el poseedor - el tiempoestá después de lo poseído - el interior- cuando debería precederlo. Este hipérbaton permite, no obstante, una más que sugerente rima entre «tiempo»y «momento/instante».

En cualquier caso, únicamente fue motivo de algún ligero comentario la traducción de «içinde», primero por un muy neutro «en» $y$

Orhan Pamuk. El lector puede encontrar varias de sus obras en prosa en español - traducidas por el autor de este artículo-, aunque no su poesía, breve pero intensa.

I2 Türk Edebiyatı Dışa Açılımı, «Apertura al exterior de la literatura turca». Es un programa de subvenciones a la traducción del Ministerio de Cultura y Turismo.

I3 «Ni estoy en el tiempo / ni por completo fuera, / sino en el flujo indivisible / de un momento extenso y monolítico».

I4 En el que participamos, aparte de Hazal Gül, İrfan Güler, Gülsevim Erhan, Pepa Baamonde, Olcay Öztunalı y el autor de este artículo. 
más tarde por «dentro», pero no la posición relativa de ambos adverbios en sus respectivos versos. La traducción inicial de «Ne içindeyim zamanın / Ne de büsbütün dışında» era «Ni estoy en el tiempo / ni fuera por completo» y la final fue «Ni estoy dentro del tiempo / ni fuera por completo».

Surge ahora un punto estrechamente relacionado con la dispositio. ¿Valdría la pena mantener la rima o procurar una parecida? Lo cual nos lleva al segundo: ¿Es deseable conseguir unos metros regulares? ¿O es mejor centrarse en los posibles efectos retóricos de la elocutio mediante figuras como este paralelismo? $\mathrm{O}$, dicho en términos de Holmes, ¿vamos a seguir una forma analógica u orgánica en nuestro metapoema? En este caso, no hay duda de que se prefirió una solución orgánica y que la traducción fue adquiriendo forma según se realizaba.

Llegada la hora de «sopesar las valencias», que diría Francis R. Jones, parece que se tuvo más en cuenta la antítesis "dentro/fuera» que posibles analogías formales. Sin embargo, visto el resultado final, esto no es del todo cierto. Manteniendo el paralelismo formal — con la elipsis del verbo «estar» en el segundo verso del original-, se consigue una rima asonante entre «tiempo» $y$ «completo», posiblemente involuntaria, pero que hasta cierto punto sirve para justificar la que acabó apareciendo en los versos tercero y cuarto. Asimismo, en nuestro metapoema los dos primeros versos son heptasílabos, lo que no combina mal con el último, endecasílabo, ya que ambos tipos pertenecen a lo que Esteban Torre llama «versos de ritmo endecasilábico» (Torre, 2000/2014: 75-77).

Desde el punto de vista de la compositio mucho más radical fue la versión de los versos tercero y cuarto ${ }^{15}$ ya planteada en la primera

15 «Yekpâre, geniş bir anın / parçalanmaz akışında»

traducción porque intercambian su posición: el tercero pasa a ser cuarto y viceversa. Existen para ello razones sintácticas porque los sustantivos que fundamentan ambos versos («instante/ an» $\mathrm{y}$ «flujo/akı̧̧») - y que en turco aparecen al final de cada uno de ellos- establecen una relación de posesión que en turco y en español se expresa exactamente al revés. En español también se ha añadido la adversativa «sino» por motivos de cohesión ya que en caso contrario probablemente habría sido necesario repetir el verbo — «estoy»—. ${ }^{16}$ Curiosamente, en ningún momento del debate se discutió la conveniencia o no de este intercambio de posiciones, sino que casi todo se centró en la traducción de «yekpâre» y en buscar alternativas a «monolítico».

Las posibilidades de colocación se complicaron aún más en este poema, pues no son solo los dos últimos versos del cuarteto —en español sería un serventesio- los que intercambian posiciones, sino también los adjetivos que califican a «instante». Mientras en el original dice: «yekpâre, geniş bir anın», en español se cambia el orden de los adjetivos «de un extenso [genis] y monolítico [yekpâre] instante [an]». No vamos a entrar en la discusión de si estos adjetivos deberían preceder o seguir al sustantivo. Aunque en español el significado no sea exactamente el mismo según la posición con respecto al sustantivo, vamos a considerar que es poéticamente aceptable que lo precedan, sobre todo por la rima que se crea entre «incesante» e «instante».

Pero, ¿por qué cambiar la posición de los adjetivos entre ellos si no afecta a la métrica

\footnotetext{
* «Monolítico, extenso un momento-de / indivisible flujoen». Ya en la primera versión (de Hazal Gül) se invierte en orden de los versos: «Sino en el flujo indivisible / de un momento extenso y monolítico». La versión final fue: «Sino en el flujo incesante / de un extenso y monolítico instante».

I6 Aunque la eliminación de «sino» nos proporciona un heptasílabo.
} 
ni a la rima, que depende de «instante»? Si el verso fuera "de un monolítico y extenso instante» nos encontraríamos con un perfecto endecasílabo sáfico desde el punto de vista del ritmo - es decir, con acentos en las sílabas cuarta, octava y décima-, pero con una pausa muy incómoda después de la sexta sílaba al ser «monolítico» palabra esdrújula, mientras que el ritmo habitual en español ante pausa es el de sílaba acentuada-sílaba no acentuada (un troqueo). La versión final - «de un extenso y monolítico instante»- tampoco resuelve del todo el problema; como endecasílabo es más imperfecto incluso que el otro, con los acentos marcados en tercera, séptima y décima sílabas. $\mathrm{El}$ acento en tercera podría haberle permitido ser un endecasílabo melódico si hubiera tenido otro en la sexta, pero de nuevo «monolítico» lo desestabiliza porque introduce un acento muy marcado en la séptima sílaba. Sin embargo, es precisamente este acento tan poco conveniente el que puede darnos una clave. En efecto, al estar en la séptima sílaba coincide exactamente con el principal del octosílabo que le precede, lo cual, además, justifica ese verso tan poco adecuado por lo general a los endecasílabos ${ }^{17} \mathrm{y}$ que rompe con los dos primeros, heptasílabos. Se podría contraargumentar que este octosílabo — «sino en el flujo incesante»— también tiene un acento en la cuarta que armonizaría con la posibilidad "de un monolítico y extenso...», pero especialmente en los versos cortos - de arte menor- el acento dominante es el último.

En cualquier caso, vemos que nos encontramos con dos fenómenos de cambio de orden que podrían ser considerados esencialmente distintos. Uno, el intercambio entre los versos tercero y cuarto, se debe a motivos fundamen-

17 Podríamos decir que tiene una valencia muy baja con los endecasílabos. talmente sintácticos. Por el contrario, el otro, la transposición entre «yekpâre, geniş» $\mathrm{y}$ «extenso y monolítico» parece deberse más bien a motivos rítmicos. Algo parecido a esto último ocurre con el hecho de que en el verso tercero nos encontremos con una estructura «sustantivo + adjetivo» y en el siguiente las posiciones se inviertan «adjetivo/adjetivo + sustantivo» simplemente para crear una rima entre «incesante» e «instante». Estas decisiones se deben ante todo a motivos puramente elocucionales, por lo que desde un punto de vista retórico corresponderían a la compositio.

No obstante, también es perfectamente lícito preguntarse por el aspecto semántico de este cambio de orden entre los adjetivos. ¿Afecta de algún modo al significado del verso la posición que tengan en su interior? La pregunta no carece de sentido porque desde el punto de vista que estamos adoptando, si esta especie de «quiasmo interlingüístico»-0 «inversión», tal y como la entiende Vázquez Ayora (vid. Hurtado Albir, 20or: 263) — supone una alteración en el significado nos encontraríamos más bien ante un fenómeno que podríamos analizar como correspondiente a la dispositio. Ya en la primera traducción de Hazal Gül («de un momento extenso y monolítico»), «yekpâre» pierde el lugar privilegiado al inicio del verso - lo que podríamos llamar posición temática versal, si es que existe tal cosa - aunque ocupa la otra posición importante, al final. Por fin, fue Pepa Baamonde quien sugirió invertir las posiciones relativas de sustantivo y adjetivos («de un extenso y monolítico instante») y crear así la rima con «incesante», palabra también propuesta por ella. Si el motivo de esta inversión fue realmente crear una rima - en suma, un efecto sonoro-, nos encontramos, sin duda, ante una operación de compositio; es decir, un cambio en el orden sintáctico por razones estilísticas en el nivel de 
la elocutio. En cambio, si se debiera a una diferencia en cuanto a la consideración semántica de los elementos que obligara a alterar su orden, nos encontraríamos más bien en el de la dispositio. El asunto no es baladí porque «yekpâre» es un término fundamental en la concepción bergsoniana del tiempo de Tanpinar: el tiempo no es una acumulación de instantes sino un continuo indivisible y en ese sentido podríamos afirmar que no es lo mismo «un instante monolítico» que «un monolítico instante».

¿Cómo habría que interpretar, pues, hechos como la inversión del tercer y cuarto versos, necesaria desde el punto de vista de la sintaxis española? ¿Como algo impuesto por el orden natural de la frase y un tanto decorativo y, por lo tanto, limitado al nivel de la elocutio? ¿O como una alteración en la disposición de los elementos que componen el discurso? Habría que tener muy presente, como hemos dicho, la globalidad del texto y huir de un análisis y una traducción verso por verso para situarnos realmente en este nivel de la dispositio. Pero también habría que tener en cuenta un factor más, muy notable en la traducción de lenguas como el turco al español: ¿hasta qué punto son similares los esquemas tema-rema en dos idiomas tan dispares sintácticamente? Es indudable que las técnicas de progresividad del texto no son exactamente iguales en ambas lenguas y que afectan a la disposición de los contenidos, por lo que habría que andarse con mucho cuidado para que cambios en el nivel sintáctico o más general de la elocutio no acabaran afectando también a la disposición de los contenidos (dispositio) desde el punto de vista de la totalidad del texto.

\section{CONCLUSIÓN}

Como ya decía Holmes, el mantenimiento de las formas métricas o el empleo de otras equi-

valentes no es precisamente la tendencia actual en la traducción de poesía y se emplea más en clases de lengua que en ediciones pensadas para el público general, a no ser que exista una voluntad particular de estilo como ocurre con clásicos con una larga tradición de traducciones, por ejemplo, Catulo o Shakespeare. Hoy en día no serían aceptables, a no ser como experimento académico, las libertades de los renacentistas españoles con los autores latinos e italianos por mucho que adaptaran una forma nueva a la literatura de su propia lengua. El grado cero en traducción actualmente está muy claro y es el contenido del texto original, de cada uno de sus formantes, para ser más exactos - y aquí volvemos al ejemplo del soneto-.

Sin embargo, igual de mal considerada, o peor, está la traducción en prosa de originales en verso. Por lo general, se intenta mantener una estructura en versos con la misma partición que en el original. ¿Por qué? Muy posiblemente porque, sobre todo en composiciones en verso libre, el verso se toma como unidad de traducción y cuando no coincide con pausas sintácticas se asume que es por motivos estilísticos que se tratan de mantener. Entonces, ¿por qué no hacer lo mismo con, por ejemplo, la rima? En talleres como el mencionado, en debates, mesas redondas, charlas, etc., puede observarse que la preocupación principal de muchos traductores de poesía es transmitir con la mayor riqueza posible el significado de determinados términos clave; es decir, todo se mantiene dentro de los límites de la inventio como creadora de los límites referenciales del poeta y del poema concreto. Pero como muchas veces es difícil que coincidan lo que Stefano Arduini llama los «campos retóricos» ${ }^{18}$

I8 Arduini, 2000; vid. especialmente los capítulos «El campo retórico», págs. 45-57, muy relacionado con la traducción, $\mathrm{y}$ «Construir mundos, intellectio y estructura del campo retórico», págs. 59-7I. 
de las lenguas en juego, el debate se estanca en la discusión de detalles sobre las posibles connotaciones de palabras muy determinadas, a menudo perdiendo de vista el contexto textual.

Es precisamente lo que ocurrió en el debate que hemos puesto como ejemplo con la palabra «yekpâre», que se convirtió en el centro de atención en detrimento de todo lo demás. Sin embargo, y por seguir citando a Arduini, «[e] locutio y dispositio no son aislables y objeto de un tratamiento independiente [con respecto a la inventio]» (Arduini, 2000: 22). Dicho de otra manera, el texto es un todo en el que las partes forman un sistema unitario. En palabras de García Berrio y Albaladejo:

La macroestructura textual o estructura profunda textual es aquella parte del texto que forman las relaciones subyacentes del producto lingüístico que sobrepasan el ámbito oracional; por su parte, la microestructura textual es el conjunto formado por las estructuras de superficie de las oraciones del texto y por las estructuras subyacentes de dichas oraciones. Según el esquema de las operaciones retóricas, la macroestructura sería resultado de la inventio y de la dispositio, mientras que la microestructura lo sería de la elocutio. («Estructura composicional. Macroestructuras», 1983: 143)

Una aproximación a la traducción de poesía que entienda la retórica como disciplina que se ocupa del texto en un sentido global debería tener en cuenta todos los aspectos, no únicamente los más relacionados con la inventio. Por otra parte, teniendo presente esta visión del hecho retórico cabría deducir que el componente transformacional, es decir la traducción en sí, tendría lugar a partir de las macroestructuras de la inventio y la dispositio para producir una elocutio con unas microestructuras diferentes del original. Sin embargo, la traducción también ocurre en la inventio y en la dispositio, esta última incluso en el nivel microtextual — como en el ejemplo de la inversión del orden adjetivos-sustantivo- Como dice Arduini: «la traducción presupone un modelo integrado en que no quede excluido ninguno de los aspectos retórico-textuales» (2000: 159).

Por lo tanto, no solo parece adecuado sino también conveniente intentar aplicar la terminología y las categorías de una retórica entendida como disciplina general del texto a una actividad generadora de textos como es la traducción. Dicho de otra forma, aunque no lleguemos a considerar la traducción como un género literario, ${ }^{19}$ sí que podemos verla y tratarla como un procedimiento retórico, especialmente en textos como los poéticos, muchas veces sometidos a unos esquemas preestablecidos de gran rigidez pero que, quizá por eso mismo, permiten tanta libertad a la hora de traducirlos.

RECIBIDO EN ENERO DE 2018 ACEPTADO EN JULIO DE 2018 VERSIÓN FINAL DE AGOSTO DE 2018

\section{BIBLIOGRAFÍA}

Albaladejo Mayordomo, Tomás (i988-i989): «Semántica y sintaxis del texto retórico: inventio, dispositio y partes orationis», E.L.U.A. Estudios de Lingüistica Universidad de Alicante, no. 5, 9-15.

Arduini, Stefano (2000): Prolegómenos a una teoría general de las figuras, Murcia: Universidad de Murcia, Servicio de Publicaciones.

Barthes, Roland (1964) : «Rhétorique de l'image», Communications, 4, 40-5I.

- (1970): «La retórica antigua. Prontuario», en La aventura semiológica, trad. de Ramón Alcalde, Barcelona: Paidós, $2^{a}$ edición española,I993.

19 Como proponía Eduardo Mendoza en 2006 en las XIV Jornadas en torno a la traducción literaria de Tarazona (vid. «Conferencia» en Vasos Comunicantes, n ${ }^{\circ} 6$, invierno de 2006, págs. 2I-22). 
Bassnett, Susan (1980): Translation Studies, 3rd edition, London: Routledge 2002.

Carpintero, Rafael (20I7): «Back to Basics: Transfer as a Metaphorical Process», Litera, no. 27 (I), I-2I.

Cirlot, Juan Eduardo (ig68): Diccionario de símbolos, Barcelona: Labor, $9^{\text {a }}$ ed., I992.

Connolly, David (I998): «Poetry Translation», en Mona Baker (ed.): Routledge Encyclopedia of Translation Studies, London and New York: Routledge, 200I.

García Berrio, Antonio (I984): «Retórica como ciencia de la expresividad. Presupuestos para una retórica general», E.L.U.A. Estudios de Lingüística Universidad de Alicante, $\mathrm{n}^{\circ}$ 2, 7-59.

García Berrio, Antonio y Tomás Albaladejo Mayordomo (I983): «Estructura composicional. Macroestructuras», E.L.U.A. Estudios de Lingüistica Universidad de Alicante, $\mathrm{n}^{\circ}$ I, I27-180.

Grupo $\mu$ (1977) : Rhétorique de la poésie. Lecture linéaire, lecture tabulaire, Paris: Éditions du Seuil, I990.

- (1992): Tratado del signo visual. Para una retórica de la imagen, trad. de Manuel Talens Carmona, Madrid: Cátedra, I993.

- (I970): Retórica general, trad. de Juan Victorio, Barcelona: Paidós, I987 (edición española revisada).

Hjelmslev, Louis (1943): Prolegómenos a una teoría del lenguaje, trad. de José Luis Díaz de Liaño, Madrid: Gredos, I97I.

Holmes, James S. (1970): «Forms of Verse Translation and the Translation of Verse Form», en James S. Holmes (ed.): The Nature of Translation:
Essays on the Theory and Practice of Literary Translation, The Hague-Paris: Mouton.

Hurtado Albir, Amparo (200I): Traducción y traductología. Introducción a la traductología, Madrid: Cátedra.

Jаковson, Roman (1958): «Linguistics and Poetics», en Thomas A. Sebeok (ed.): Style in Language, Cambridge, Mass.: Technology Press of Massachusetts Institute of Technology; New York \& London : John Wiley \& Sons,I96o.

Jones, Francis R. (1989): «On Aboriginal Sufferance: A Process Model of Poetic Translating», Target, vol. I, $\mathrm{n}^{\circ}$ 2, I83-I99.

Lefevere, André (1975): Translating Poetry: Seven Strategies and a Blueprint, Amsterdam: Van Gorcum.

- (I992): Traducción, reescritura y la manipulación del canon literario, trad. de $\mathrm{M}^{\mathrm{a}}$ del Carmen África Vidal y Román Álvarez, Salamanca: Colegio de España, 1997.

Lindgren, Marcia H., Life Blumberg y Joshua Langseth (2010): «From Literal to Literary: A Translation Project for Latin Poetry Classes», Teaching Classical Languages, vol. I, no 2, primavera, IO9-I37.

Morris, Charles (1938): Fundamentos de la teoría de los signos, trad. de Rafael Grasa, Barcelona: Paidós, I985.

Torre, Esteban (2000/20I4): Métrica española comparada, Sevilla: Universidad de Sevilla.

- (2003): «Sílabas y acentos. Fundamentos fonéticos y fonológicos del ritmo», Rhythmica, vol. I, no. I, pp. 273-301. 